\title{
NORM ATTAINING OPERATORS AND SIMULTANEOUSLY CONTINUOUS RETRACTIONS
}

\author{
JERRY JOHNSON AND JOHN WOLFE ${ }^{1}$
}

\begin{abstract}
A compact metric space $S$ is constructed and it is shown that there is a bounded linear operator $T: L^{1}[0,1] \rightarrow C(S)$ which cannot be approximated by a norm attaining operator. Also it is established that there does not exist a retract of $L^{\infty}[0,1]$ onto its unit ball which is simultaneously weak* continuous and norm uniformly continuous.
\end{abstract}

1. Introduction. Let $X$ and $Y$ be Banach spaces. A linear operator $T: X \rightarrow$ $Y$ is called norm attaining if there is an $x \in X$ with $\|x\|=1$ and $\|T x\|=\|T\|$. Schachermayer in [5] has constructed an example of an operator $T_{0}: L^{1}[0,1] \rightarrow$ $C[0,1]$ such that if $\left\|T-T_{0}\right\| \leq \frac{1}{2}$ and $\|T\| \leq 1$ then $T$ is not norm attaining. This is the first example of a pair of classical Banach spaces $X, Y$ such that the norm attaining operators from $X$ to $Y$ are not dense. Unfortunately, Schachermayer's example is very lengthy and intricate.

In this note we provide a simple example of a compact metric space $S$ and an operator $T_{0}: L^{1}[0,1] \rightarrow C(S)$ such that if $\left\|T-T_{0}\right\| \leq \frac{1}{2}$ and $\|T\| \leq 1$, then $T$ is not norm attaining. This is Corollary 2. The ideas are definitely inspired by Schachermayer's construction.

This example will be a simple corollary of Theorem 1 . Another corollary provides an answer to a question of Benyamini concerning retractions on dual spaces. By a selection argument there is always a weak* continuous retraction of the dual of any separable Banach space onto its unit ball (the retraction $x \rightarrow x /\|x\|$ for $\|x\| \geq 1$ is norm continuous but never weak* continuous except in the finite dimensional case). Benyamini [1] proved an embedding result about separable $C(K)$ spaces by establishing the existence of a retraction of the dual of $C[0,1]$ onto its unit ball which is simultaneously weak ${ }^{*}$ continuous and norm uniformly continuous. Then in [2] Benyamini goes on to show that such simultaneously continuous retractions exist in the dual of $l^{p}$ or $L^{p}[0,1]$ for $1<p<\infty$. We will show that no such retraction is possible in the dual of $L^{1}[0,1]$. This is Corollary 3.

Notation is standard (cf. [3]), all subsets of $[0,1]$ below are assumed to be Borel and $m$ denotes Lebesgue measure.

Let $S_{0}=\left\{\sum_{i=1}^{n}\left(1-2^{-i}\right) \chi_{D_{i}} \mid D_{i} \subset[0,1]\right.$ are disjoint and $\left.m D_{i}<2^{-1}\right\}$. Throughout this note $S$ will denote the weak ${ }^{*}$ closure of $S_{0}$ in $L^{\infty}[0,1]=L^{1}[0,1]^{*}$. Thus, $S$ is a compact metric space whose essential property for our purposes is contained in the following.

Received by the editors February 2, 1982.

1980 Mathematics Subject Classification. Primary 46B25; Secondary 47A99.

${ }^{1}$ Research supported in part by NSF grant MCS8002544. 
THEOREM 1. Let $\psi: S \rightarrow L^{\infty}$ be weak* continuous and suppose, for each $s \in S$, $\|\psi(s)-s\|_{\infty} \leq \frac{1}{2}$ and $\|\psi(s)\|_{\infty} \leq 1$. Then for each $s \in S$ and each $k \geq 2$

$$
m\left\{\omega \in[0,1] \mid \psi(s)(\omega) \geq 1-2^{-k-2}\right\} \leq 16 \cdot 2^{-k} .
$$

The applications of this result stem from the following observation. Consider the points $g \in L^{\infty}[0,1]$ such that $\|g\|=1$ and $m\{\omega:|g(\omega)|=\|g\|=1\}>0$. Such points $g$ are dense in the surface of the ball in $L^{\infty}$ and lie arbitrarily close to $S$, but they do not satisfy the distributional condition of Theorem 1. It is somewhat surprising that it is impossible to move the points in $S$ around the unit ball of $L^{\infty}$ in a weak ${ }^{*}$ continuous manner (with no point moved more than norm distance $\frac{1}{2}$ ) by a map $\psi$ such that one of the many nearby points $g$ as above is in the range of $\psi$.

This theorem will be proved in $\S 3$. We now state the two applications which will be derived from Thoerem 1 in $\S 2$.

Corollary 2. Define $T_{0}: L^{1}[0,1] \rightarrow C(S)$ by $T_{0} f(s)=\int_{0}^{1} f(\omega) s(\omega) d \omega$ for $f \in$ $L^{1}$ and $s \in S$. If $T: L^{1}[0,1] \rightarrow C(S)$ is a linear operator with $\left\|T-T_{0}\right\| \leq \frac{1}{2}$ and $\|T\| \leq 1$ then $T$ is not norm attaining.

COROLLARY 3. There is no weak * continuous and norm uniformly continuous retraction of $L^{\infty}[0,1]$ onto its unit ball.

ACKNOWLEDGEMENT. The connection between a counterexample as in Corollary 2 and Corollary 3 was observed independently by the second author, W. Schachermayer and C. Stagall during a lecture by Y. Benyamini delivered at Oberwolfach in the summer of 1981 .

2. Proofs of Corollaries 1 and 2. To see Theorem 1 implies Corollary 2 observe the well-known fact (cf. [3 and 4]) that $T: L^{1}[0,1] \rightarrow C(S)$ corresponds canonically to a weak* continuous function $\psi: S \rightarrow L^{\infty}[0,1]$ and that $T$ is norm attaining if and only if there is a point $s \in S$ such that $\|\psi(s)\|=\|T\|=\|\psi\|$ and $\psi(s)$ is norm attaining as a functional on $L^{1}[0,1]$; i.e. there is a set $E$ so that $m E>0$ and $|\psi(s)|=\|\psi\|$ on $E$. Now, the identity map: $S \rightarrow L^{\infty}[0,1]$ represents $T_{0}$ and so $\|\psi(s)-s\| \leq \frac{1}{2}$. Hence Theorem 1 implies the desired result.

Now, to see that Theorem 1 yields Corollary 3 suppose such a retraction $\varphi$ exists. Choose $\delta>0$ such that $\|f-g\|<\delta$ implies $\|\varphi(f)-\varphi(g)\|<\frac{1}{2}$. Let $s_{0} \in S$ be of norm 1 (e.g. $\left.s_{0}=\sum_{n=1}^{\infty}\left(1-2^{-n}\right) \chi_{D_{n}}, D_{n}=\left(2^{-n-1}, 2^{-n}\right)\right)$. There is a function $g \in L^{\infty}[0,1]$ with $g(\omega)=\|g\|=1$ on a set of positive measure and $\left\|g-s_{0}\right\|<\delta$. Define $\psi: S \rightarrow L^{\infty}[0,1]$ by $\psi(s)=\varphi\left(s+g-s_{0}\right)$. Then $\|\psi(s)-s\|=$ $\left\|\varphi\left(s+g-s_{0}\right)-\varphi(s)\right\| \leq \frac{1}{2}$ since $\left\|s+g-s_{0}-s\right\|<\delta$. Also $\|\psi\| \leq 1$ and $\psi\left(s_{0}\right)=$ $\varphi(g)=g$. This contradicts Theorem 1 .

3. Proof of Theorem 1. In order to prove Theorem 1 we need the following:

LEMMA 1. Suppose $s=\sum_{i=1}^{n}\left(1-2^{-i}\right) \psi_{D_{i}} \in S_{0}$. Then $\left\|\left.\psi(s)\right|_{D_{i}}\right\|_{\infty} \leq 1-2^{-i-2}$ for each $i=1, \ldots, n$.

Before we prove Lemma 1, let us show how Theorem 1 follows from it.

To do this first observe that for $s=\sum_{i=1}^{n}\left(1-2^{-i}\right) \psi_{D_{i}} \in S_{0}$ and for $k \leq 2$,

$$
m\left\{\omega \mid \psi(s)(\omega) \geq 1-2^{-k}\right\} \leq 8 \cdot 2^{-k} \text {. }
$$


For, if $A=\left\{\omega \mid \psi(s)(\omega) \geq 1-2^{-k}\right\}$, then by Lemma $1, m\left(A \cap D_{i}\right)=0$ when $1-$ $2^{-i-2}<1-2^{-k}$; i.e. when $i<k-2$. Also, $m\left(A \sim \bigcup_{i=1}^{n} D_{i}\right)=0$ since $\|\psi(s)-s\| \leq \frac{1}{2}$ and $k \geq 2$. Thus $m A \leq \sum_{i=k-2}^{n} m D_{i} \leq \sum_{i=k-2}^{\infty} 2^{-i}=8 \cdot 2^{-k}$, and (0) holds. Now if the conclusion of Theorem 1 fails, then for some $s \in S$ and $k \geq 2$, there is a set $E$ with $m E=16 \cdot 2^{-k}$ and $\int_{E} \psi(s) \geq m E\left(1-2^{-k-2}\right)$. Given any $\epsilon>0$, we may choose $s_{1} \in S_{0}$ such that $\int_{E} \psi(s) \leq \int_{E} \psi\left(s_{1}\right)+\epsilon$, since $S_{0}$ is weak dense in $S$. Using (0) we may break $E$ into two sets $E_{1}$ and $E_{2}$ with $m E_{1}=m E_{2}=\frac{1}{2} m E=8 \cdot 2^{-k}$ so that $\psi\left(s_{1}\right)<1-2^{-k}$ on $E_{2}$. Thus

$$
\begin{aligned}
m E\left(1-2^{-k-2}\right) & \leq \int_{E} \psi(s) \leq \int_{E_{1}} \psi\left(s_{1}\right)+\int_{E_{2}} \psi\left(s_{1}\right)+\epsilon \\
& \leq m E_{1}+\left(1-2^{-k}\right) m E_{2}+\epsilon=m E\left(1-2^{-k-1}\right)+\epsilon
\end{aligned}
$$

Since $\epsilon>0$ is arbitrary, we have a contradiction and Theorem 1 is proved.

In order to prove Lemma 1 , we require the following simple observations:

Suppose $s=\sum_{i=1}^{n} \alpha_{i} \chi_{D_{i}}$, with $0<\alpha_{i}<1$ and the $D_{i}$ 's disjoint. Let $A$ be a fixed subset of some $D_{i}$ and let $\Pi=\left\{A_{1}, \ldots, A_{k}\right\}$ be a partition of $A$. Choose $C_{j} \subset A_{j}$ with $m C_{j}=2 \alpha_{i} m A_{j} /\left(i+\alpha_{i}\right)$ and define

$$
T_{\Pi} s=\sum_{j=1}^{k} \frac{1+\alpha_{1}}{2} \chi_{C_{j}}+s \chi_{\tilde{A}}
$$

The following are easy to check:

(1) $\int_{A_{j}} s=\int_{A_{j}}\left(1+\alpha_{i}\right) \chi_{C_{j}} / 2$.

(2) $\int_{F} s=\int_{F} T_{\Pi} s$ if $f \cap A=\varnothing$ or if $F$ is a union of sets from $\Pi$. (This follows from (1).)

(3) $m\left(\left\{\omega \mid T_{\Pi} s(\omega)=0\right\} \cap A\right)=\left(1-\alpha_{i}\right) m A /\left(1+\alpha_{i}\right)$.

(4) Weak ${ }^{*}-\lim _{\Pi} T_{\Pi} s=s$ where the limit is taken over partitions of $A$ ordered by refinement.

Property (4) follows from (2). For, if $E \subset[0,1]$ and $\Pi$ is a refinement of $\{A \cap$ $E, A \sim E\}$, then

$$
\begin{aligned}
\int_{E} T_{\Pi} s & =\int_{E \sim A} T_{\Pi} s+\int_{E \cap A} T_{\Pi} s+\int_{A \sim E} T_{\Pi} s \\
& =\int_{E \sim A} s+\int_{E \cap A} s+\int_{A \sim E} s=\int_{E} s .
\end{aligned}
$$

Now, assume $s \in S_{0}$ and Lemma 1 fails. Then there is a set $A \subset D_{i}$ such that $m A>0,(1 / m A) \int_{A} \psi(s)>1-2^{-i-2}$ and $m A+m D_{i+1}<2^{-i-1}$. But if $\Pi$ is a partition of $A$ and if $E=\left\{\omega \mid T_{\Pi} s(\omega)=0\right\} \cap A$, then $T_{\Pi} s \in S_{0}$ and

$$
\int_{A} \psi\left(T_{\Pi} s\right)=\int_{A \sim E} \psi\left(T_{\Pi} s\right)+\int_{E} \psi\left(T_{\Pi} s\right) \leq m(A \sim E)+\frac{1}{2} m E .
$$

A simple calculation using the definition of $T_{\Pi}$ shows that $m(A \sim E)+\frac{1}{2} m E \leq$ $m A\left(1-2^{-i-2}\right)$.

Now, taking the weak* limit on $\Pi$ and using (4) we get that

$$
\int_{A} \psi(s) \leq m A\left(1-2^{-i-2}\right)
$$

a contradiction.

This completes the proof of Lemma 1 . 


\section{REFERENCES}

1. Y. Benyamini, Small into-isomorphisms between spaces of continuous functions, preprint.

2. __ Simultaneously continuous retractions on the unit wall of a Banach space, preprint.

3. N. Dunford and J. T. Schwartz, Linear operators, Part I, Interscience, New York, 1958.

4. J. Johnson and J. Wolfe, Norm attaining operators, Studia Math. 65 (1979), 7-19.

5. W. Schachermayer, Norm attaining operators on some classical Banach spaces, Pacific J. Math. (to appear).

Department of Mathematics, OKLahoma State University, Stillwater, OKLAHOMA 74078 